\title{
LA TERAPIA FOTODINÁMICA EN UROLOGÍA. MECANISMOS DE ACCIÓN BIOLÓGICOS Y PATOLÓGICOS
}

\author{
Pilar González-Peramato', Javier Regadera² y Ángeles Juarranz³.
}

'Departamento de Anatomía Patológica. Hospital Universitario La Paz Madrid

2Departamento de Anatomía, Histología y Neurociencia. Facultad de Medicina. Universidad Autónoma de Madrid.

${ }^{3}$ Departamento de Biología. Facultad de Ciencias. Universidad Autónoma de Madrid. España.

\begin{abstract}
Resumen.- La Terapia Fotodinámica (TFD) es una modalidad terapéutica mínimamente invasiva y de extraordinaria utilidad. En Urología, la TFD, es muy útil y puede ser aplicada mediante endoscopia o directamente, habiéndose obtenido excelentes resultados en el diagnóstico y tratamiento de lesiones tumorales de vejiga, en el tratamiento de carcinoma de próstata y de sus recidivas y en el tratamiento de lesiones dermatológicas precancerosas y tumorales del pene.
\end{abstract}

La TFD se fundamenta en el uso de compuestos fotosensibilizadores (FSs) que se acumulan selectivamente en los tejidos tumorales. La irradiación posterior de éstos tejidos con luz de longitud de onda apropiada lgeneralmente de la región del rojo del espectro visible $\lambda \geq 600 \mathrm{~nm}$ ) produce la formación de especies reactivas de oxígeno (ROS) con efectos citotóxicos,
Pilar González-Peramato Departamento de Anatomía Patológica Hospital Universitario La Paz Paseo de la Castellana 261 28046 Madrid. (España). mpgonzalez.hulp@salud.madrid.org que conducen a la muerte selectiva de las células neoplásicas y en consecuencia a la regresión del tumor. La principal ventaja de la TFD es que el daño celular queda restringido a la zona irradiada, con la consiguiente disminución de efectos secundarios sobre los tejidos sanos próximos al tumor, al contrario de lo que ocurre con otras terapias convencionales de algunos tumores del tracto urinario. Además, la TFD puede utilizarse en combinación con radioterapia y quimioterapia.

Palabras clave: Terapia fotodinámica. Cáncer de vejiga. Cáncer de próstata. Cáncer de pene. Láser.

Summary.- Photodynamic Therapy (FDT) is a minimally invasive therapeutic modality extraordinarily useful. In urology, FDT is very useful and may be applied through endoscopes or directly, with excellent results obtained for the diagnosis and treatment of bladder tumors, in the treatment of prostate cancer and its recurrences, and in the treatment of dermatological premalignant lesions and carcinomas of the penis.

FDT is founded on the use of photosensitizing products which selectively accumulate in fumor tissues. The irradiation of these tissues with a proper wavelength light lgenerally in the red region of the visible spectrum $\lambda \geq 600 \mathrm{~nm}$ ) produces the formation of oxygen reactive species with cytotoxic effects leading to selective death of neoplastic cells, and tumor regression. The main advantage of FDT is the restriction of cellular damage to the irradiation area, with the associated decrease of secondary effects on healthy tissues near the tumor, on the contrary to what happen with other conventional therapies for some tumors of the urinary tract. Moreover, FDT may be used in combination with radiotherapy and chemotherapy. 
Keywords: Photodynamic therapy. Bladder cancer. Prostate cancer. Penis cancer. Laser.

\section{INTRODUCCIÓN}

El tratamiento con luz de lesiones premalignas y malignas del tracto urinario ha demostrado ser de utilidad, tanto por su eficacia terapéutica como también por su facilidad de manejo clínico y, además, por su mínima o ausente morbilidad. Dentro de las aplicaciones de los diferentes tipos de luz en Urología, se encuentra la combinación de la luz con compuestos fotosensibilizadores (FSs), lo cual ha dado origen a la novedosa técnica de Terapia Fotodinámica (TFD). Mediante esta modalidad de tratamiento, se consigue una destrucción rápida de las células tumorales, sin efectos dañinos sobre células y tejidos normales adyacentes.

Las primeras fuentes de luz utilizadas en TFD, eran fuentes de luz incoherentes, con diferentes filtros que podían variar selectivamente la longitud de onda, pero ofrecían numerosas desventajas (dificultad para controlar la dosis de luz, efecto térmico y baja intensidad). Por ello, con el avance tecnológico en la TFD, se han mejorado las fuentes de luz, como las lámparas de LED (Light-emiting diodes) capaces de generar luz de elevada energía y de longitudes de onda específicas, adecuadas a las necesidades de cada FS, al igual que los láseres. Estos últimos constituyen una de las fuentes mas utilizadas en TFD (1).

La TFD ha sido aprobada para el tratamiento tanto curativo como paliativo de algunos tipos de cáncer y de lesiones premalignas y también para el tratamiento de la degeneración de la mácula óptica con la edad (Tabla I). Actualmente está siendo principalmente utilizada en Dermatología para el tratamiento de lesiones premalignas, queratosis actínicas y malignas -carcinomas basocelulares, la enfermedad de Bowen, el melanoma amelanótico, linfomas de células T, "etc"-. por la aplicación directa con sistemas de iluminación de luz visible o de laser. También, la TFD se puede aplicar mediante procedimientos endoscópicos utilizando fibras ópticas, para el tratamiento de lesiones neoplásicas de los epitelios de revestimiento del tracto digestivo, respiratorio, urinario y del cérvix uterino $(1,2)$. A dichas aplicaciones terapéuticas, es necesario mencionar además, el empleo de la TFD como herramienta diagnóstica, ya que la fluorescencia producida por los FSs (3), especialmente por la Protoporfirina IX (PpIX), está siendo utilizada para la detección temprana de lesiones pretumorales o tumorales, principalmente de piel, pulmón y vejiga.

La TFD se basa en la capacidad que poseen ciertos compuestos con características fotosensibilizadoras de acumularse de forma preferente en las células y tejidos tumorales. El compuesto FS, en presencia de oxígeno, es

\section{TABLA I. FOTOSENSIBILIZADORES ACTUALMENTE APROBADOS PARA TRATAMIENTO DE TUMORES MALIGNOS Y PREMALIGNOS.}

\begin{tabular}{|l|l|l|l|}
\hline Fotosensibilizador & \multicolumn{1}{|c|}{$\begin{array}{c}\text { Grupo Químico } \\
\text { (Nombre Comercial) }\end{array}$} & \multicolumn{1}{|c|}{ Indicaciones } & \multicolumn{1}{|c|}{ Paises } \\
\hline $\begin{array}{l}\text { Derivado hematoporfirínico, } \\
\text { Polihematoporfirina }\end{array}$ & Porfirinas (Photofrin®) & $\begin{array}{l}\text { Esófago de Barrett } \\
\text { Displasia cervical } \\
\text { Cáncer cervical } \\
\text { Cáncer de pulmón } \\
\text { Cáncer gastrico superficial } \\
\text { Adenocarcinoma de Esófago } \\
\text { Cáncer superficial de vejiga }\end{array}$ & $\begin{array}{l}\text { Estados Unidos, Unión Euro- } \\
\text { pea, Canadá, Rusia, Japón, } \\
\text { etc. }\end{array}$ \\
\hline $\begin{array}{l}\text { Tetra(m-hidoxifenil) clorina } \\
\text { mTHPC, Temoporfin }\end{array}$ & Clorinas (Foscan®) & $\begin{array}{l}\text { Tratamiento paliativo de cáncer } \\
\text { de cabeza y cuello }\end{array}$ & Unión Europea \\
\hline $\begin{array}{l}\text { Ácido aminolevulínico } \\
\text { (ALA)* }\end{array}$ & Porfirinas (Levulan®) & Queratosis actínica & Estados Unidos \\
\hline $\begin{array}{l}\text { Metil ester-aminolevulínico } \\
\text { (MAL)* }\end{array}$ & Porfirinas (Metvix®) & $\begin{array}{l}\text { Queratosis actínica y } \\
\text { Carcinomas basocelulares }\end{array}$ & Unión Europea \\
\hline $\begin{array}{l}\text { Hexil ester-aminolevulínico } \\
\text { (HAL)* }\end{array}$ & Porfirinas (Hexvix®) & $\begin{array}{l}\text { Diagnóstico por fluorescencia } \\
\text { de tumores de vejiga }\end{array}$ & Unión Europea \\
\hline
\end{tabular}

Además de los compuestos indicados, el derivado monoácido de benzoporfirina (BPS-MA) o verteporfina (Visudyne®) está siendo utilizado para la degeneración de la mácula con la edad en oftalmología. * ALA, MAL y HAL son precursores del compuesto fotoactivo protoporfirina IX (PpIX). 
activado con luz de longitud de onda apropiada, normalmente de la región del rojo del espectro de luz visible (> $600 \mathrm{~nm})$, dado que la penetración de esta luz en los tejidos es mayor. El FS excitado transfiere su energía al oxígeno molecular $\left(\mathrm{O}_{2}\right)$ para originar oxígeno singlete $\left({ }^{\prime} \mathrm{O}_{2}\right)$, y otras especies reactivas de oxígeno (reactive oxygen species, ROS), de modo que se desencadena una cascada bioquímica que conducen a la muerte selectiva de las células tumorales y en consecuencia de la regresión del tumor. Por lo tanto, tres agentes: el compuesto FS, la luz y el $\mathrm{O}_{2}$, que por ellos mismos no son tóxicos, son los responsables de la muerte de las células tumorales $(4,5)$.

Dentro de las principales ventajas de la acción terapéutica de la TFD, sobre otros tratamientos de cáncer más convencionales, se encuentran:

1) su escasa toxicidad sistémica, debido a la preferente acumulación del compuesto FS en células tumorales,

2) su habilidad para destruir selectivamente a las células tumorales, ya que su efecto citotóxico sólo ocurre tras la irradiación con luz del área tumoral en la que se ha acumulado el FS,

3) la posibilidad de aplicar la TFD sola o en combinación con otros tratamientos del cáncer, incluidos la quimioterapia, la radioterapia y la inmunoterapia, y

4) la ausencia de efectos mutagénicos, permitiendo además mantener indemnes los tejidos sanos adyacentes. Además, el compuesto FS tiene la ventaja de poder ser administrado por vía sistémica o mediante aplicación tópica, en función de la localización del tumor $(1,3)$.

\section{Bases fotoquímicas y compuestos fotosensibilizadores}

En líneas generales, la energía del compuesto FS excitado por la luz puede ser disipada en forma de calor, de fluorescencia, o interactúan con el oxígeno molecular $\left(\mathrm{O}_{2}\right)$ para de este modo formar:

1) especies reactivas de oxígeno (ROS), tales como el anión

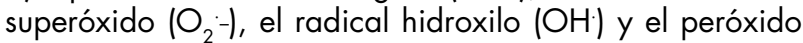
de hidrógeno $\left(\mathrm{H}_{2} \mathrm{O}_{2}\right)$, esto es, una reacción fotoquímica de tipo I, o bien

2) originar oxígeno singlete, $\left({ }^{1} \mathrm{O}_{2}\right)$, mediante una reacción fotoquímica de tipo II (Figura 1). Aunque el mecanismo preciso no se conoce, se asume que la mayor parte de los FSs empleados en TFD actúan principalmente mediante reacciones de tipo II, siendo por tanto el ${ }^{1} \mathrm{O}_{2}$ el principal responsable de la muerte de las células tumorales. No obstante, la contribución de ambos tipos de reacciones a la muerte celular depende de varios factores, incluyendo la naturaleza del FS, su localización subcelular y la presencia de $\mathrm{O}_{2}$.

En general, en condiciones hipóxicas sucede principalmente una reacción fotodinámica de tipo l; mientras que en medios oxigenados, prevalecen las reacciones de tipo II. Además, es necesario señalar que las ROS presentan una vida media muy corta; cabe mencionar que la vida media del ${ }^{1} \mathrm{O}_{2}$ en sistemas biológicos es tan sólo de 0.04 $\mu s$, siendo su radio de acción de $0.02 \mu \mathrm{m}$ (5). Por ello, solo las células y tejidos que estén muy cerca de los lugares de generación de ROS serán los inicialmente dañados. El compuesto FS es efectivo cuando es capaz de introducirse en las células tumorales, pudiéndose afectar diversas dianas y organelas subcelulares -mitocondrias, lisosomas, membrana plasmática, retículo endoplásmico, aparato de Golgi- por el proceso fotodinámico. Estas alteraciones desencadenan la muerte de las células tumorales por apoptosis, necrosis u otros mecanismos de muerte celular (6-12) (Figura 2).

El segundo componente de la TFD es la luz. En general, los compuestos FSs que se emplean en TFD presentan

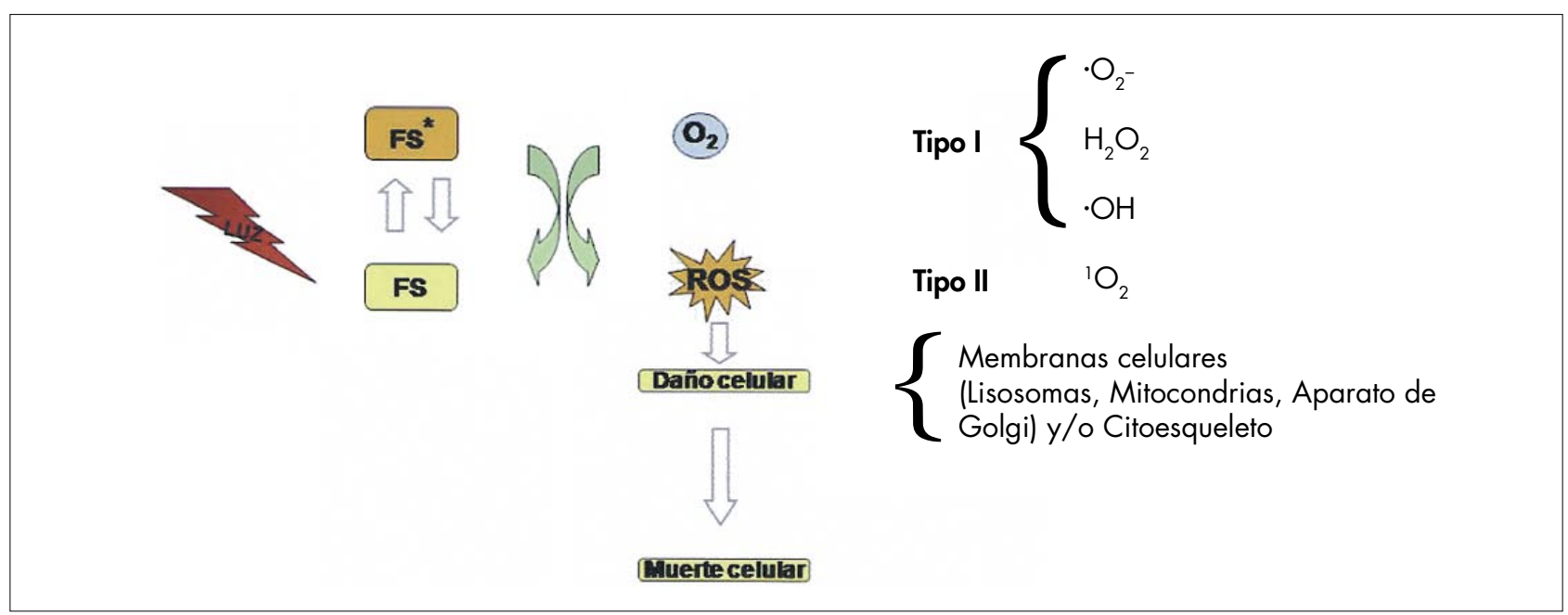

FIGURA 1. Mecanismos de respuesta biológica celular producidos por sustancias fotosensibilizantes: reacción fotoquímica de tipo Il que provoca la muerte celular. 


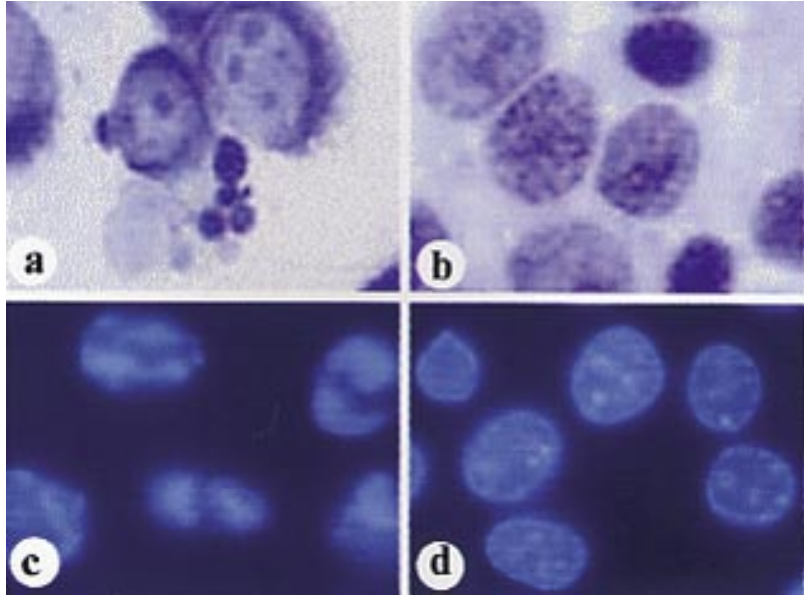

FIGURA 2. Daño celular producido por la aplicación de terapia fotodinámica en cultivos celulares. A y C: daño de la cromatina nuclear caraterística de apoptosis, B y D: cambios nucleares típico de lesiones de necrosis. A y B: tinción de Giemsa observado con un microscopio de campo claro. Cy D: tinción nuclear "daping" visto con fluorescencia.

una elevada absorción en la región roja del espectro de luz visible $(600-800 \mathrm{~nm})$. Ello, se relaciona con la alta capacidad de penetración de la luz, en función de la longitud de onda, en el interior de los tejidos. Así, la luz de 500-600nm penetra aproximadamente unos $4 \mathrm{~mm}$, mientras que puede llegar hasta una profundidad de $8 \mathrm{~mm}$ cuando las longitudes de onda son de 600-800nm (13). Pero además, el empleo de la fibra óptica ha marcado una revolución en el tratamiento de las neoplasias, sobre todo en aquellas lesiones observables endoscópicamente. De este modo, el tratamiento endoscópico mediante TFD de pequeñas lesiones displásicas y neoplásicas permite obtener resultados muy satisfactorios y ausencia de efectos secundarios en el tratamiento de lesiones precoces y cánceres avanzados de pulmón, estómago, esófago, cérvix y vejiga, sobre todo mediante la aplicación de Photofrin® y luz roja (4).

Los compuestos naturales y sintéticos que presentan propiedades fotosensibilizadoras potencialmente aplicables en TFD son de naturaleza química muy variada. Entre ellos, caben destacar principalmente: las porfirinas (principalmente el derivado hematoporfirínico, $\mathrm{HpD}$ y su forma comercial purificada Photofrin $®$ - el cual fue el primer FS aprobado para el tratamiento de cánceres humanos en Canadá y Estados Unidos-, las benzoporfirinas, las ftalocianinas, las clorinas, las purpurinas, las bacterioclorinas y las verdinas, y otros muchos compuesto más de uso aún no completamente estandarizado (1,14-16). Además, también se ha empleado con gran efectividad la Protoporfirina IX (PpIX), la cual es sintetizada endógenamente a partir de la pro-droga o pro-fotosensibilizador ácido amino 5-levulínico (ALA) en la ruta de síntesis del grupo hemo. (Figura 3). En esta ruta, intervienen también otras moléculas, como la hemoglobina, los citocromos y la vitamina $B_{12}(17,18)$.

La naturaleza hidrofílica del ALA puede limitar, sin embargo, su capacidad de penetración a través de las membranas celulares, incluso en el tratamiento de lesiones muy superficiales. Para obviar esta situación, se están investigando derivados menos hidrofílicos de ALA, como son los esteres hidrocarbonados, con una mayor capacidad de penetración en las lesiones neoplásicas profundas, destacando su derivado metil (MAL, Metvix®) o el hexil ALA (HAL, Hexvix®) (18-20).

De entre los diferentes compuestos FSs que se utilizan en TFD son, sin duda, el ALA (Levulan $®$ ) y sus derivados los que presentan mayor eficacia, empleándose, tanto para el diagnóstico, como para el tratamiento de lesiones benignas cutáneas -acné y psoriasis- y para neoplasias superficiales -queratosis actínicas, enfermedad de Bowen, carcinomas epidermoides y basocelulares de piel-. El Me-

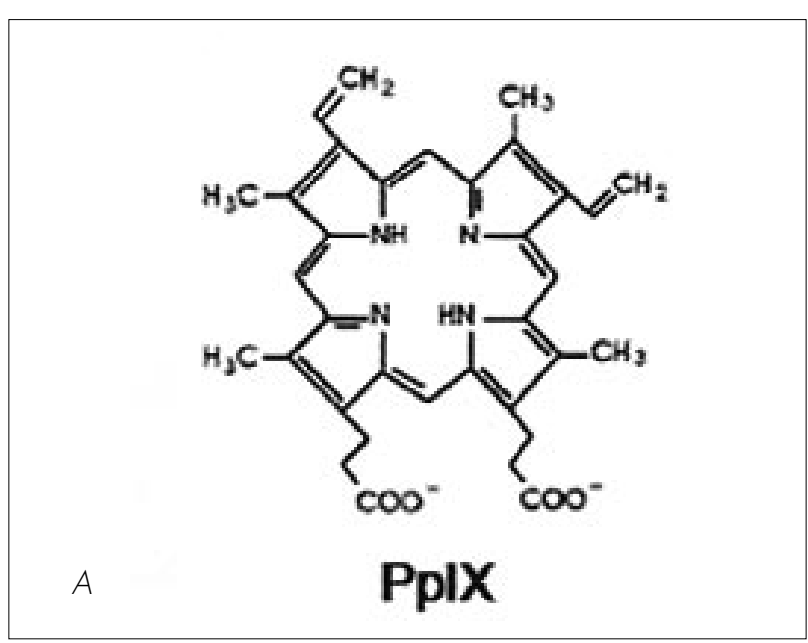

FIGURA 3. A: Fórmula del fotosensibilizante protoporfina IX

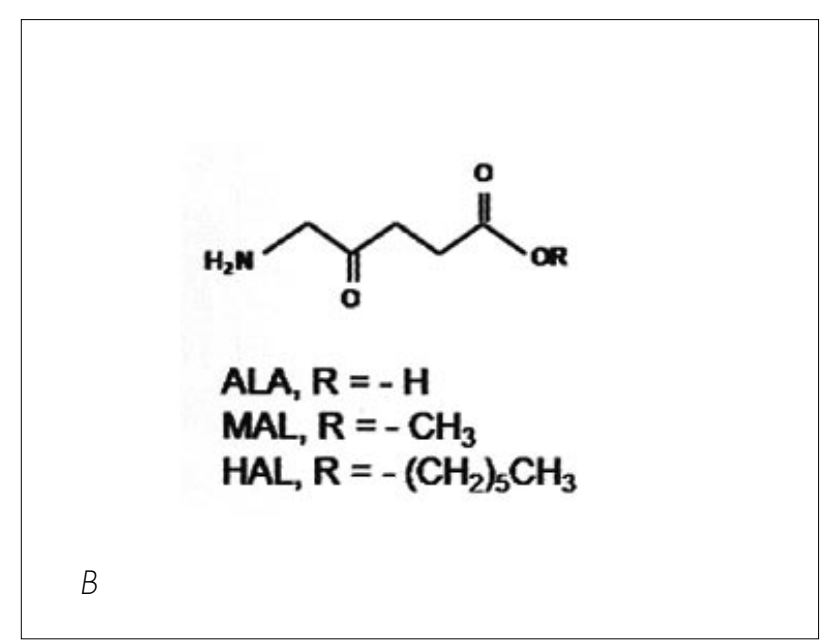

B: Fórmula del fotosensibilizante ácido amino-levulínico. 
tvix® es el único compuesto aprobado en Europa para el tratamiento de queratosis actínica y carcinomas basocelulares $(2,17,21)$.

En tumores de órganos profundos, y en concreto en los tumores del tracto urinario, la aplicación de TFD usando como producto FS Hexvix ${ }^{\circledR}$, ha mejorado las tasas de detección de lesiones planas y papilares de pequeño tamaño de la vejiga y ya ha sido aprobado para su uso como método diagnóstico en 26 países europeos $(4,22)$.

\section{Mecanismos de acción de la TFD}

Los mecanismos por los que el $10^{2}$ y otras ROS contribuyen a la destrucción del tumor mediante TFD son principalmente tres:

1) Daño celular directo,

2) Daño celular indirecto, por alteraciones en la vascularización tumoral y

\section{3) Activación del sistema inmune (3).}

La contribución relativa de cada uno de los tres mecanismos depende de varios factores como son: la propia naturaleza del FS, su localización y concentración en el tumor durante el proceso de irradiación, el tipo de tumor y su vascularización (13,23-30).

Los compuestos FSs, cuando se administran sistémicamente, se acumulan preferentemente en los tejidos tumorales, lo cual es lógico si se tiene presente los altos índices de proliferación de las células neoplásicas. Pero además, la acumulación de los FSs en las células tumorales está relacionada con su grado de hidrofobicidad, por lo que el uso de liposomas como vehículos para transportar los FS en sangre, permite no sólo administrar ciertos FSs, sino además mejorar la liberación de los mismos en los órganos diana. Los FSs hidrofóbicos o vehiculizados en liposomas son transportados por las lipoproteínas séricas de baja densidad (LDL), y, dado que las células tumorales presentan un elevado número de receptores para dichas proteínas, se consigue de este modo una mayor penetración del FS en el tumor (31).

La concentración de los FSs varía en los diferentes órganos, pero también dependiendo de las situaciones particulares de cada paciente: las mismas condiciones terapéuticas pueden determinar concentraciones distintas de compuesto FS; por ello, es importante ajustar el volumen de emisión de fluorescencia en cada órgano y tejido, siendo necesario establecer previamente un mapeo de distribución tridimensional del FS en cada órgano (32). Además, los niveles de FS en los tejidos deben ajustarse a la dosis de luz aplicada con el fin de reducir la variabilidad del comportamiento del compuesto FS en cada paciente y evitar consecuencias terapéuticas adversas (33).

Se deben investigar la aplicación de varias pautas de tratamiento con TFD en los diferentes tipos y localizaciones de los tumores, con el fin de valorar cómo las dosis de luz y las diferentes drogas aplicadas en distintas combinaciones incrementan la respuesta terapéutica de cada tipo de tumor, al actuar tanto sobre las células neoplásicas, como sobre la vascularización intratumoral neoformada (34). En este sentido, está completamente establecido que la TFD lesiona también indirectamente a las células tumorales a través del daño a la vascularización tumoral y, así mismo, modificando la respuesta de las células inmunocompetentes dentro de los tejidos neoplásicos $(3,13,35)$. En definitiva, la respuesta terapéutica varía de unos órganos a otros, dado que la concentración total de oxígeno tisular depende del flujo sanguíneo vascular y de la diferente difusión del oxígeno desde la sangre a los distintos tipos de tejidos.

Para que las células tumorales adquieran la capacidad de infiltración vascular y metástasis, se requiere la presencia de oxígeno y nutrientes que permitan la supervivencia de las células tumorales. Por ello, se considera en la actualidad que los vasos tumorales constituyen importantes dianas en el tratamiento tumoral. La aplicación de la TFD contribuye al daño de la vascularización del tumor, al favorecer la formación de trombos que limitan la aportación de oxígeno y la nutrición al tumor. Los compuestos FSs que ocasionan un mayor daño vascular son la HPD, y el Photofrin $尺$, los cuales, acumulados selectivamente en los vasos sanguíneos y estimulados por la luz, producen una progresiva destrucción de la vascularización tumoral (31). El daño específico e irreversible de las células endoteliales de los vasos intratumorales activa una cascada de procesos que conducen a la dilatación vascular, agregación de plaquetas, vasoconstricción, hemorragias, inflamación y alteraciones de la respuesta inmunológica locales (36-38). Estos hechos, facilitan los procesos muerte de las células tumorales, pero además, la aplicación de TFD produce lesiones histopatológicas locales asociadas, entre las que se incluyen el eritema agudo, el edema y la necrosis; en definitiva, se produce un efecto vascular hipóxico que determina una disminución del crecimiento tumoral $(3,39-41)$.

Cuando los FSs se utilizan sistémicamente, el efecto terapéutico está en función del tiempo transcurrido después de su administración y del grado de vascularización del tumor, dado que estas substancias primeramente y durante un corto periodo de tiempo se localizan dentro de los vasos sanguíneos, para después extravasarse gradualmente. Es más, habitualmente la exposición a la luz se realiza unas 3 ó 4 horas después de administrar el compuesto por vía sistémica, sin embargo para dañar los vasos sanguíneos tumorales, la exposición debería realizarse poco después de la administración del FS ya que éste, una vez administrado, tan sólo tarda unos 15 minutos en distribuirse por el plasma. (42). Pero además, es necesario tener en cuenta que muy probablemente las lesiones celulares y moleculares que provocan los FSs sobre las células tumorales y sobre la microvascularización y angiogénesis tumoral podrían quedar moduladas por tratamientos previos de radioterapia y quimioterapia del tumor primario.

En la actualidad, se están diseñando líneas de investigación que combinan protocolos de aplicación de TFD con los bien estandarizados tratamientos oncológicos 
de quimioterapia y radioterapia, previéndose en un futuro inmediato un gran potencial terapéutico de la combinación de TFD con inhibidores de microtúbulos o con la administración de compuestos antiangiogénicos. En esta línea de investigaciones fronteras aplicadas a la clínica, cabe destacar el diseño de métodos más precios de la denominada TFD intersticial, la cual se está investigando en el tratamiento de enfermedades inflamatorias y neoplásicas de órganos sólidos parenquimatosos, y concretamente en Urología se está aplicando dentro de protocolos terapéuticos del cáncer de próstata, de vejiga y de pene (43).

\section{APLICACIÓN DE LA TFD EN CÁNCER DE PRÓSTATA}

En pacientes con cáncer de próstata inicial, la TFD comenzó a aplicarse en la década de los 90 del siglo pasado (44). Los pacientes sometidos a 8-10 sesiones de TFD presentaron una disminución de la expresión de PSA (aproximadamente del 67\%). En estos casos, la resonancia magnética nuclear mostró edema y necrosis parcheada de la próstata, que solía resolverse en aproximadamente dos meses. La biopsia prostática de estos pacientes reveló que las áreas tratadas tenían sólo necrosis y fibrosis, por lo cual se concluyó que la TFD era una técnica muy útil para el tratamiento de lesiones iniciales de cáncer de próstata (45).

En la actualidad, se ha demostrado que la aplicación de la TFD, si bien permite obtener buenos resultados como tratamiento primario de pequeñas lesiones malignas prostáticas, los mejores resultados terapéuticos se consiguen en los pacientes que presentan recidivas después del tratamiento con radioterapia (46), que, como es bien sabido, constituyen un problema clínico importante, dado que las herramientas terapéuticas que quedan para poder aplicarse en estos pacientes son escasas.

En el tratamiento del cáncer de próstata -sobre todo en los tumores recidivantes- $(44,46)$ y del carcinoma transicional de vejiga (47) se están usando con buenos resultados los FSs. HPD y el Photofrin $®$. Además, en el cáncer de próstata se ha aplicado TFD usando ALA, lo que mejora sustancialmente el efecto de fotosensibilización celular y el resultado del tratamiento. Sin embargo, en algunos casos, su efecto terapéutico no es completamente efectivo; por ello, es necesario realizar estudios nuevos in vitro e in vivo que combinen ALA-TFD con drogas quimioterápicas, como pueden ser el metotrexate o los vinca alcaloides (48).

La TFD ocasiona un efecto citotóxico directo sobre las células tumorales prostáticas e indirecto sobre las mismas, por la destrucción de los vasos sanguíneos intratumorales (49). En este sentido, en pacientes con cáncer de próstata recidivante, la administración de un compuesto de la familia de las metalo texafirinas (motexafin lutetium) 3 horas antes de aplicar la luz, consiguió una reducción del flujo sanguíneo prostático de un $50 \%$ y una reducción de la concentración de hemoglobina total del 15\%; sin embargo, solo se objetivó una disminución un 3\% de la saturación total de oxígeno en la próstata después del tratamiento. Estos datos sugieren que la aplicación de esta droga y de la TFD tiene un evidente efecto antivascular en el tejido tumoral prostático (50).
En pacientes con cáncer de próstata tratados previamente con radioterapia, se ha usado como FS un derivado de paladio de una bacterioclorina (palladiumbacteriopheophorbide), cuya diana principal son los vasos intratumorales. En estos pacientes se ha encontrado una correlación positiva entre la dosis de luz aplicada y el tamaño de la zona prostática destruida, de modo que elevadas dosis de luz permitieron destruir el $70 \%$ de las zonas periféricas de la próstata; además, en estos pacientes que recibieron radioterapia y TFD no se encontraron efectos adversos importantes como incontinencia urinaria y los pacientes conservaron la potencia sexual. Además, en estos casos los resultados terapéuticos, valorados histológicamente y por resonancia magnética nuclear, fueron satisfactorios y mejores que los encontrados cuando solo se aplicó radioterapia externa sobre la próstata (51).

En el cáncer de próstata es muy importante determinar la concentración de FS y el grado de oxigenación tisular cuando se aplica TFD (52). La distribución del FS se puede analizar in vivo, mediante el uso de una fibra óptica que permita capturar la fluorescencia, y determinar la concentración de FS mediante espectroscopía de absorción o de fluorescencia. De este modo, es posible demostrar la existencia de importantes variaciones intraprostáticas de la distribución de la droga, sugiriéndose que debería realizarse una dosimetría en tiempo real, monitorizando estos valores durante la aplicación de tratamiento con TFD (52).

La administración de la luz en el cáncer de próstata se puede realizar mediante fibra óptica insertada transperinealmente con la misma plantilla que la braquiterapia. Se realizan biopsias prostáticas antes y después de la aplicación de la luz, con el fin de medir, mediante espectrofluorometría, la captación de FS (49). Con todo, se observa una heterogeneidad de los resultados en el tratamiento, siendo necesario un planteamiento terapéutico individualizado para cada paciente (49). El uso de fibras ópticas difusoras cilíndricas que se insertan perpendicularmente en una placa base permite obtener uniformidad en la cantidad de luz que llega a cada área prostática, preservando la uretra y el recto (53). En este sentido, un modelo experimental en perros ha demostrado una distribución uniforme en la próstata de la luz emitida por una fibra cilíndrica. Estos resultados indican que para poder aplicar correctamente la TFD en el cáncer de próstata, es necesario diseñar buenos programas computarizados que puedan ser aplicados en tiempo real. (54).

Tumores experimentales de próstata en ratas permiten valorar los resultados terapéuticos de la TFD (34). En estos casos, la aplicación del derivado de benzoporfirina, Verteporfin $\AA$, ocasionó cambios en la vascularización del tumor, 15 minutos después de la inyección, consiguiéndose una reducción significativa del flujo sanguíneo tumoral e incremento de la fracción de hipoxia tumoral; pero 3 horas después se revirtió el flujo sanguíneo con valores similares a los del control sano; además histológicamente se confirmó daño de la vascularización tumoral y de las células tumorales, preservándose intactos los tejidos normales prostáticos adyacentes al área tumoral (34). En ratas, los tumores de próstata tratados con derivados de la benzoporfirina, presentaron una farmacocinética diferente en áreas más 
vascularizadas y menos vascularizadas. De esta manera; por ello, el tiempo preciso de tratamiento puede depender de la particular permeabilidad de los vasos de los distintos tipos de tejidos que recibe el tratamiento de TFD (55).

En relación con los vasos sanguíneos intratumorales del carcinoma de próstata, se han investigado los mecanismos moleculares implicados en la actuación la TFD, y de modo especial, su relación con el factor de crecimiento endotelial vascular (VEGF) (56). Se ha descrito un aumento de la secreción de VEGF en cultivos de células de carcinoma de próstata (57) sometidos a TFD. Además, en roedores se ha observado daño endotelial irreversible, debido a la necrosis y apoptosis de las células tumorales, tan sólo 15 minutos después de administrar la TFD (58). Los compuestos FSs no sólo se acumulan en las mitocondrias, sino también en el citosol, provocando cambios de señalización celular que favorecen el incremento de la síntesis de VEGF. Este incremento está relacionado con los mecanismos de hipoxia celular y tisular que están mediados por el factor de hipoxia HIF-1 $\alpha$ (59). También estaría implicado el daño en las membranas celulares $(60,61)$, y, aunque no se ha demostrado claramente, podrían ocurrir cambios de la matriz extracelular adyacente al tumor (62). Por último, en los tratamientos con TFD pueden producirse alteraciones de los mecanismos de adhesión de plaquetas y formación de trombos que ocluyen los vasos sanguíneos (62) y disminución de la adhesión entre células endoteliales $(63,64)$ e incremento de la producción de citoquinas $(65)$ y de proteínas de shock térmico (66). Estos interesantes datos experimentales nos permiten sugerir que en un futuro no my lejano se podrá intentar la aplicación de la TFD en el cáncer de próstata en combinación con agentes antiangiogénicos $(57,67)$.

La aplicación de TFD no conlleva un riesgo de diseminación de células malignas en los tejidos adyacentes, y tampoco se ha encontrado acumulación tóxica de los FSs, cuando la dosis y la intensidad de luz son adecuadas; por ello, la TFD se puede repetir las veces que sea necesario, incluso en tejidos sometidos previamente a radioterapia. Todos estos datos hacen prever un futuro prometedor del uso de la TFD en los pacientes con cáncer de próstata (68), dado que permite conseguir una alta concentración del fármaco fotosensibilizante en el tejido diana tumoral. El reto de investigación actual es encontrar qué moléculas fotosensibilizadoras con especial afinidad por los tejidos prostáticos (68) y mejorar la profundidad de exposición a la luz. En la actualidad, generalmente se alcanza una profundidad de 2-3 mm aplicando luz de $630 \mathrm{~nm}$ de longitud de onda; sin embargo, cuando se utilizan $700-800 \mathrm{~nm}$, se consigue una penetración de $5-6 \mathrm{~mm}$ (69). Por último, los nuevos fármacos usados en TFD tienen un efecto terapéutico que comienza a los pocos minutos de la aplicación de la fuente lumínica, de modo que el efecto curativo sobre los tejidos se obtiene muy rápidamente.

\section{APLICACIÓN DE LA TFD EN CÁNCER DE VEJIGA}

Es bien conocida la elevada tasa de recidivas del cáncer de vejiga suponiendo un importante gasto sanitario. De hecho se ha calculado que el tratamiento de esta neoplasia es el $5^{\circ}$ más caro en coste total, pero el primero si se evalúa en gasto por paciente. Por ello, un diagnóstico más preciso permitirá un mejor manejo de los pacientes y un ahorro significativo en el tratamiento de las recidivas de estos enfermos.

En la actualidad, los FSs se están usando principalmente como una herramienta útil en el diagnóstico clínico de neoplasias vesicales -sobre todo del carcinoma in situ y de otros tumores papilares de pequeño tamaño- mediante la realización de una citoscopia con fluorescencia, tras la administración del FS. Aunque teóricamente pueden utilizarse numerosos FSs en el diagnóstico del cáncer de vejiga, en la práctica se prefieren agentes tales como el 5-ALA y sus derivados como el hexil-ALA (Hexvix®) (70), consiguiéndose un rendimiento diagnóstico superior al de la cistoscopia tradicional de luz blanca. Además, pueden ser aplicados tópicamente mediante la instilación intravesical, evitándose la prolongada fotosensibilización cutánea residual que sucede cuando se administran sistémicamente FSs como el derivado de la Hematoporfirina (HpD) (71). El ALA se acumula rápidamente en las células uroteliales, sin efectos tóxicos secundarios para el paciente, pudiendo observarse mediante fluorescencia bajo luz de excitación azul o roja (72).

En los tumores papilares de vejiga de estadios $\mathrm{T} 1$ y Ta, también la aplicación de ALA permite diagnosticar mediante citoscopía las áreas tumorales por su intensa fluorescencia roja (73). En definitiva, el ALA mejora los resultados diagnósticos, y facilita un tratamiento precoz del cáncer de vejiga y una disminución de la aparición de recidivas (74-76) y, en consecuencia, disminuyendo el enorme gasto económico que ocasiona el carcinoma vesical recidivante (71).

En el cáncer vesical recientemente se ha aplicado la TFD, habiéndose observado una destrucción completa o parcial del tumor. En estos casos, la aplicación terapéutica de la TFD está indicada para pacientes muy ancianos o con constantes vitales comprometidas que no admiten una cirugía radical.

\section{APLICACIÓN DE LA TFD EN LESIONES DEL PENE}

Puesto que la TFD está completamente admitida como tratamiento eficaz de lesiones cutáneas premalignas y malignas, también se está comenzando a aplicar como método diagnóstico y de tratamiento de tumores de la piel del pene. Entre dichas lesiones, es de destacar la eritroplasia de Queyrat, en la cual se está usando como FS tópico el derivado metilado del ALA (Metvix $®$ ), en combinación con luz roja de $630 \mathrm{~nm}$, a dosis de $37 \mathrm{~J} / \mathrm{cm}^{2}$ (77), siendo los resultados terapéuticos satisfactorios, puesto que este procedimiento tiene la ventaja de delimitar específicamente el área tumoral, facilitando un tratamiento más local y selectivo, preservando la función del pene y obteniéndose resultados estéticos muy satisfactorios (78).

La TFD también es útil en el tratamiento del carcinoma de pene, asegurando una mayor destrucción del tejido tumoral que el conseguido por la cirugía conserva- 
dora. Es más, la TFD ha demostrado ser efectiva como tratamiento en varios tipos de lesiones cutáneas malignas del pene, incluidas el carcinoma basocelular, la enfermedad de Bowen y el carcinoma epidermoide (79).

\section{CONCLUSIÓN}

En la TFD actualmente se está empleando Metvix ${ }^{\circledR}$ para el diagnóstico y tratamiento de lesiones cutáneas de pene y Hexvix ${ }^{\circledR}$ para el diagnóstico de lesiones vesicales. Estas aplicaciones unidas a los excelentes resultados obtenidos en el tratamiento de pacientes con cáncer de próstata recidivante, hacen que la TFD sea una excelente alternativa terapéutica; bien aplicada sóla, o en combinación con terapias convencionales. La TFD podrá ser ampliamente utilizada en un futuro inmediato, tanto para el diagnóstico como para el tratamiento de lesiones tumorales en Urología.

\section{BIBLIOGRAFÍA y LECTURAS RECOMENDADAS ("lectura de interés $\mathrm{y}^{* *}$ lectura fundamental)}

1. PERVAIZ, S.; OLIVO, M.; "Art and science of photodynamic therapy”. Clin. Exp. Pharmacol. Physiol., 33: $551,2006$.

**2. JUARRANZ, A.; JAÉN, P.; SANZ-RODRÍGUEZ, F. y COLS.: "Photodynamic therapy of cancer. Basic principles and applications". Clin. Transl. Oncol. 10:148, 2008.

**3. DOLMANS, D.E.; FUKUMURA, D.; JAIN, R.K.: "Photodynamic therapy for cancer". Nat. Rev. Cancer, 3: 380, 2003.

4. JUZENIENE, A.; PENG, Q.; MOAN, J.: "Milestones in the development of photodynamic therapy and fluorescence diagnosis". Photochem. Photobiol. Sci., 6: 1234, 2007.

5. MOAN, J.; BERG, K.: "The photodegradation of porphyrins in cells can be used to estimate the lifetime of singlet oxygen". Photochem. Photobiol., 53: 549, 1991.

6. KOENIG, F.; MCGOVERN, F.J.; LARNE, R., y cols: "Diagnosis of bladder carcinoma using protoporphyrin IX fluorescence induced by 5-aminolaevulinic acid". BJU Int, 83; 129-135, 1999

7. BAUMGARTNER, R.; HUBER, R.M.; SCHULZ, H. y cols: "Inhalation of 5-aminolevulinic acid: a new technique for fluorescence detection of early stage lung cancer". J Photochem Photobiol B, 36:169-174 1996.

8. LEUNIG, A.; RICK, K.; STEPP, H. y cols: "Fluorescence imaging and spectroscopy of 5-aminolevulinic acid induced protoporphyrin IX for the detection of neoplastic lesions in the oral cavity". Am. J. Surg., 172: 674-677, 1996.

9. EKER, C.; MONTÁN, S.; JARAMILLO, E. y cols: "Clinical spectral characterisation of colonic mucosal lesions using autofluorescence and delta aminolevulinic acid sensitisation". Gut, 44:511-518, 1999.

10. LANGE, N.; JICHLINSKI, P.; ZELLWEGER, M. y cols: "Photodetection of early human bladder cancer based on the fluorescence of 5-aminolaevulinic acid hexylester-induced protoporphyrin IX: A pilot study".
Br. J. Cancer, 80: 185, 1999.

11. UEHLINGER, P.; ZELLWEGER, M.; WAGNIERES, G. y cols: "5-Aminolevulinic acid and its derivatives: physical chemical properties and protoporphyrin IX formation in cultured cells". J. Photochem. Photobiol. B, 54: 72, 2000

*12. UEHLINGER, P.; ZELLWEGER, M.; WAGNIÈRES, G. y cols: "Tumor-secreted vascular permeability factor/vascular endothelial growth factor influences photosensitizer uptake". Cancer Res. 53: 153, 1993.

13. DOUGHERTY, T.J.; GOMER, C.J.; HENDERSON, B.W. y cols: "Photodynamic therapy". J. Natl. Cancer Inst., 90: 889,1998.

14. BROWN, S.B.; MELLISH, K.J.: "Verteporfin: a milestone in opthalmology and photodynamic therapy". Expert Opin. Pharmacother., 2: 351, 2001.

15. DETTY, M.R.; GIBSON, S.L.; WAGNER, S.J.: "Current clinical and preclinical photosensitizers for use in photodynamic therapy". J. Med. Chem., 47: 3897, 2004.

16. STOCKERT, J.C.; CANETE, M.; JUARRANZ, A. y cols: "Porphycenes: facts and prospects in photodynamic therapy of cancer". Curr. Med. Chem., 14: 997, 2007.

17. BABILAS ,P.; LANDTHALER, M.; SZEIMIES, R.M.: "Photodynamic therapy in dermatology". Eur. J. Dermatol., 16: 340, 2006.

18. CASAS, A.; BATLLE, A.: "Rational design of 5-aminolevulinic acid derivatives aimed at improving photodynamic therapy". Curr. Med. Chem. Anticancer Agents,. 2: 465, 2002.

19. GAULLIER, J.M.; BERG, K.; PENG, Q. y cols: "Use of 5-aminolevulinic acid esters to improve photodynamic therapy on cells in culture2. Cancer Res., 57: 14811486, 1997.

20. LOPEZ, R.F.; LANGE, N.; GUY, R. y cols: "Photodynamic therapy of skin cancer: controlled drug delivery of 5-ALA and its esters". Adv. Drug. Deliv. Rev., 56: 77, 2004.

21. BLUME, J.E.; OSEROFF, A.R.: "Aminolevulinic acid photodynamic therapy for skin cancers" Dermatol. Clin., 25:5, 2007.

22. SCHMIDBAUER, J.; WITJES, F.; SCHMELLER, N. y cols: "Improved detection of urothelial carcinoma in situ with hexaminolevulinate fluorescence cystoscopy". J. Urol., 171: 135, 2004.

23. KIM, H.R.; LUO, Y.; LI, G. y cols: "Enhanced apoptotic response to photodynamic therapy after bcl-2 transfection". Cancer Res., 59: 3429, 1999.

24. AGARWAL, M.L.; LARKIN, H.E.; ZAIDI, S.I. y cols: "Phospholipase activation triggers apoptosis in photosensitized mouse lymphoma cells". Cancer Res., 53: 5897, 1993.

25. AGOSTINIS, P.; ASSEFA, Z.; VANTIEGHEM, A. y cols: "Apoptotic and anti-apoptotic signalling pathways induced by photodynamic therapy with hypericin". Adv. Enzyme Regul., 40: 157, 2000.

26. XUE, L.; HE, J.; OLEINICK, N.L.: "Promotion of photodynamic therapy-induced apoptosis by stress kinases". Cell Death Differ.; 6: 855, 1999.

27. XUE, L.Y.; QIU, Y.; HE, J. y cols: "Etk/Bmx, a PHdomain containing tyrosine kinase, protects prostate cancer cells from apoptosis induced by photodynamic 
therapy or thapsigargin”. Oncogene, 18: 3391-3398, 1999.

28. XUE, L.; HE, J.; OLEINICK, N.L.: "Rapid tyrosine phosphorylation of HS1 in the response of mouse lymphoma L5178YR cells to photodynamic treatment sensitized by the phthalocyanine Pc 4". Photochem. Photobiol., 66: 105, 1997.

29. GOMER, C.J.; RYTER, S.W.; FERRARIO, A. y cols: "Photodynamic therapy-mediated oxidative stress can induce expression of heat shock proteins". Cancer Res., 56: 2355, 1996.

30. GOMER ,C.J.; FERRARIO, A.; RUCKER, N.: "Glucose regulated protein induction and cellular resistance to oxidative stress mediated by porphyrin photosensitization”. Cancer Res., 51: 6574, 1991.

*31. CHEN, B.; POGUE, B.W.; HASAN, T.: "Liposomal delivery of photosensitising agents". Expert Opin. Drug. Deliv., 2: 477, 2005.

**32. FINLAY, J.C.; ZHU, T.C.; DIMOFTE, A. y cols: "Interstitial fluorescence spectroscopy in the human prostate during motexafin lutetium-mediated photodynamic therapy". Photochem. Photobiol., 82: 1270, 2006.

33. ZHOU, X.; POGUE, B.W.; CHEN, B. y cols: "Pretreatment photosensitizer dosimetry reduces variation in tumor response". Int. J. Radiat. Oncol. Biol. Phys., 15; 64:1211, 2006.

34. CHEN, B.; POGUE, B.W.; HOOPES P.J. y cols: "Combining vascular and cellular targeting regimens enhances the efficacy of photodynamic therapy. Int. J. Radiat. Oncol. Biol. Phys., 61: 1216, 2005.

35. SOLBAN, N.; RIZVI, I.; HASAN, T.: "Targeted photodynamic therapy". Lasers Surg. Med., 38: 522, 2006.

36. ROBERTS, W.G.; HASAN, T.: "Role of neovasculature and vascular permeability on the tumor retention of photodynamic agents". Cancer Res., 52:924,1992.

37. POGUE, B.W.; REDMOND, R.W.; TRIVEDI, N. ycols: "Photophysical properties of tin ethyl etiopurpurin I (SnET2) and tin octaethylbenzochlorin (SnOEBC) in solution and bound to albumin". Photochem. Photobiol., 68: 809, 1998.

38. AHMAD, N.; MUKHTAR, H.: "Mechanism of photodynamic therapy-induced cell death". Methods Enzymol., 319: 342, 2000.

39. GOMER, C.J., FERRARIO, A.; LUNA, M. y cols: "Photodynamic therapy: combined modality approaches targeting the tumor microenvironment". Lasers Surg. Med., 38: 516, 2006.

**40. CASTANO, A.P.; MROZ, P.; HAMBLIN, M.R.: "Photodynamic therapy and anti-tumour immunity". Nat. Rev. Cancer, 6: 535, 2006.

41. TOZER, G.M.; KANTHOU, C; BAGULEY, B.C.: "Disrupting tumour blood vessels". Nat. Rev. Cancer, 5:423-435, 2005.

42. ICHIKAWA, K.; HIKITA, T.; MAEDA, N. y cols: "Antiangiogenic photodynamic therapy (PDT) by using long-circulating liposomes modified with peptide specific to angiogenic vessels". Biochim Biophys. Acta., 1669:69, 2005.

43. DU, K.L.; MICK, R.; BUSCH, T.M. y cols.: "Preliminary results of interstitial motexafin lutetium-mediated PDT for prostate cancer". Lasers Surg. Med., 38: 427, 2006

44. WINDAHL, T.; ANDERSSON, S.O.; LOFGREN, L.:
"Photodynamic therapy of localised prostatic cancer". Lancet, 336: 1139, 1990.

45. MOORE, C.M.; NATHAN, T.R.; LEES, W.R. y cols.: "Photodynamic therapy using meso tetra hydroxy phenyl chlorin (mTHPC) in early prostate cancer". Lasers Surg. Med., 38: 356, 2006.

46. NATHAN, T.R.; WHITELAW, D.E.; CHANG, S.C. y cols.: "Photodynamic therapy for prostate cancer recurrence after radiotherapy: a phase I study". J Urol. 168: 1427, 2002.

**47. MUSCHTER, R.: "Photodynamic therapy: a new approach to prostate cancer". Curr. Urol. Rep., 4: 221, 2003.

48. SINHA, A.K.; ANAND, S.; ORTEL, B.J. y cols.: "Methotrexate used in combination with aminolaevulinic acid for photodynamic killing of prostate cancer cells". Br. J. Cancer, 95: 485, 2006.

49. VERIGOS, K.; STRIPP, D.C.; MICK, R. y cols.: “Updated results of a phase I trial of motexafin lutetiummediated interstitial photodynamic therapy in patients with locally recurrent prostate cancer". J. Environ. Pathol. Toxicol. Oncol. 25: 373, 2006.

50. YU, G.; DURDURAN, T.; ZHOU, C. y cols: "Realtime in situ monitoring of human prostate photodynamic therapy with diffuse light. Photochem Photobiol, 82: 1279, 2006.

*51. TRACHTENBERG, J.; BOGAARDS, A., WEERSINK, R.A. y cols.: "Vascular targeted photodynamic therapy with palladium-bacteriopheophorbide photosensitizer for recurrent prostate cancer following definitive radiation therapy: assessment of safety and treatment response". J. Urol., 178: 1974, 2007.

52. ZHU, T.C.; FINLAY, J.C.; HAHN, S.M.: "Determination of the distribution of light, optical properties, drug concentration, and tissue oxygenation in-vivo in human prostate during motexafin lutetium-mediated photodynamic therapy". J. Photochem. Photobiol B., 79: 231, 2005 .

53. ALTSCHULER, M.D.; ZHU, T.C.; LI, J.; y cols.: "Optimized interstitial PDT prostate treatment planning with the Cimmino feasibility algorithm". Med. Phys., 32: 3524, 2005

54. JANKUN,J.;KECK,R.W.;SKRZYPCZAK-JANKUN, E. y cols.: "Diverse optical characteristic of the prostate and light delivery system: implications for computer modelling of prostatic photodynamic therapy". BJU Int., 95: 1237, 2005.

55. ZHOU, X., POGUE, B.W.; CHEN, B. y cols.: "Analysis of effective molecular diffusion rates for verteporfin in subcutaneous versus orthotopic Dunning prostate tumors". Photochem. Photobiol., 79: 323, 2004.

**56. SOLBAN, N.; PA ${ }^{\circ}$, S.K.; ALOK, S.K. y cols.: "Mechanistic Investigation and Implications of Photodynamic Therapy Induction of Vascular Endothelial Growth Factor in Prostate Cancer". Cancer Res., 66: 5633, 2006.

57. FERRARIO, A.; VON TIEHL, K.F.; RUCKER, N. y cols.: "Antiangiogenic treatment enhances photodynamic therapy responsiveness in a mouse mammary carcinoma". Cancer Res., 60:4066, 2000.

58. NOWIS, D.; MAKOWSKI, M.; STOKLOSA, T. y cols.: "Direct tumor damage mechanisms of photodynamic therapy". Acta Biochim. Pol., 52: 339, 2005. 
59. FORSYTHE, J.A.; JIANG, B.H.; IYER, N.V. y cols.: "Activation of vascular endothelial growth factor gene transcription byhy poxia-inducible factor 1 . Mol. Cell. Biol. 16: 4604, 1996.

60. SPECHT, K.G.; RODGERS, M.A.: "Plasma membrane depolarization and calcium influx during cell injuryby photodynamic action”. Biochim. Biophys. Acta 1070: 60, 1991.

61. KUNZ, L.; STARK, G.: "Photofrin II sensitized modifications of ion transport across the plasma membrane of an epithelial cell line. I. Electrical measurements at the whole-cell level". J. Membr. Biol., 166: 179, 1998.

62. CHEN, B., POGUE, B.W.; LUNA, J.M. y cols.: "Tumor Vascular Permeabilization by Vascular-Targeting Photosensitization: Effects,Mechanism, and Therapeutic Implications". Clin. Cancer Res. 12: 917, 2006.

63. RUNNELS, J.M.; CHEN, N.; ORTEL, B. y cols.: "BPDMA-mediated photosensitization in vitro and in vivo: cellular adhesion and $\mathrm{h} 1$ integrinexpression in ovarian cancer cells". Br. J. Cancer, 80: 946, 1999.

64. ROUSSET, N.; VONARX, V.; ELE`OUET, S. y cols.: "Effects of photodynamic therapy on adhesion molecules and metastasis. J. Photochem. Photobiol. B. Biol. 52: $65,1999$.

*65. DOUGHERTY, T.J.; MARCUS, S.L.: "Photodynamic therapy". Eur. J. Cancer, 28A: 1734, 1992.

66. LUNA, M.C.; FERRARIO, A.; WONG, S. y cols.: "Photodynamic therapy-mediated oxidative stress as a molecular switch for the temporal expression of genes ligated to the human heat shock promoter". Cancer Res., 60: 1637, 2000.

67. ZHOU, Q.; OLIVO, M.; LYE, K.Y. y cols.: "Enhancing the therapeutic responsiveness of photodynamic therapy with the antiangiogenic agents SU5416 and SU6668 in murine nasopharyngeal carcinoma models". Cancer Chemother. Pharmacol. 56: 569, 2005.

**68. RAMÍREZ BACKHAUS, M.; TRASSIERRA VILLA, M.; VERA DONOSO, C.D. y cols.: "Terapia fotodinámica en el cáncer de próstata localizado". Actas Urol. Esp., 31: 633, 2007.

69. SVAASAND, L.O.; ELLINGSEN, R.: "Optical properties of human brain". Photochem. Photobiol., 38: 293, 1983.
70. KRAMMER, B.; PLAETZER, K.: "ALA and its clinical impact, from bench to bedside". Photochem. Photobiol. Sci. 7:283, 2008.

*71. WITJES, J.A.; DOUGLASS, J.: "The role of hexaminolevulinate fluorescence cystoscopy in bladder cancer". Nat. Clin. Pract. Urol., 4: 542, 2007.

72. SCHMIDBAUER, J.; MARBERGER, M.: "Recent developments in fluorescence cystoscopy: do novel agents bring a benefit?". Curr. Opin. Urol., 17: 347, 2007.

73. GROSSMAN, H.B.; GOMELLA, L.; FRADET, Y; y PC B302/01 STUDY GROUP. "A phase III, multicenter comparison of hexaminolevulinate fluorescence cystoscopy and white light cystoscopy for the detection of superficial papillary lesions in patients with bladder cancer". J. Urol., 178: 62, 2007.

*74. FRADET, Y.; GROSSMAN, H.B.; GOMELLA, L.; PC B302/01 STUDY GROUP y cols.: "A comparison of hexaminolevulinate fluorescence cystoscopy and white light cystoscopy for the detection of carcinoma in situ in patients with bladder cancer: a phase III, multicenter study". J. Urol., 178: 68, 2007.

75. DENZINGER, S.; BURGER, M.; WALTER, B. y cols.: "Clinically relevant reduction in risk of recurrence of superficial bladder cancer using 5-aminolevulinic acidinduced fluorescence diagnosis: 8-year results of prospective randomized study". Urology, 69: 675, 2007.

76. BURGER, M.; ZAAK, D.; STIEF, C.G. y cols.: "Photodynamic diagnostics and noninvasive bladder cancer: is it cost-effective in long-term application? A Germanybased cost analysis". Eur. Urol., 52: 142, 2007.

77. LEE, M.R.; RYMAN, W.: "Erythroplasia of Queyrat treated with topical methyl aminolevulinate photodynamic therapy". Australas. J. Dermatol., 46: 196, 2005.

78. STABLES, G.I.; STRINGER, M.R.; ROBINSON, D.J. y cols.: "Erythroplasia of Queyrat treated by topical aminolaevulinic acid photodynamic therapy". Br. J. Dermatol.,140: 514, 1999.

79. HARTH, Y.; HIRSHOWITZ, B.; KAPLAN, B.: "Modified topical photodynamic therapy of superficial skin tumors, utilizing aminolevulinic acid, penetration enhancers, red light, and hyperthermia". Dermatol. Surg., 24:723, 1998. 